\title{
Cytoskeletal Integrators: The Spectrin Superfamily
}

\section{Ronald K.H. Liem}

Department of Pathology and Cell Biology, Columbia University Medical Center, New York, New York 10032

Correspondence: rkl2@cumc.columbia.edu

\section{SUMMARY}

This review discusses the spectrin superfamily of proteins that function to connect cytoskeletal elements to each other, the cell membrane, and the nucleus. The signature domain is the spectrin repeat, a 106-122-amino-acid segment comprising three $\alpha$-helices. $\alpha$-actinin is considered to be the ancestral protein and functions to cross-link actin filaments. It then evolved to generate spectrin and dystrophin that function to link the actin cytoskeleton to the cell membrane, as well as the spectraplakins and plakins that link cytoskeletal elements to each other and to junctional complexes. A final class comprises the nesprins, which are able to bind to the nuclear membrane. This review discusses the domain organization of the various spectrin family members, their roles in protein-protein interactions, and their roles in disease, as determined from mutations, and it also describes the functional roles of the family members as determined from null phenotypes.

\section{Outline}

1 Introduction

$2 \alpha$-actinin

3 Spectrins

4 Dystrophin and utrophin
5 Spectraplakins and plakins

6 Nesprins

7 Concluding remarks

References

Editors: Thomas D. Pollard and Robert D. Goldman

Additional Perspectives on The Cytoskeleton available at www.cshperspectives.org

Copyright $\odot 2016$ Cold Spring Harbor Laboratory Press; all rights reserved; doi: 10.1101/cshperspect.a018259 


\section{INTRODUCTION}

Cytoskeletal elements, such as microtubules, microfilaments, and intermediate filaments, are linked to each other, to the cell membrane, and to the nucleus by a class of cytoskeletal integrators. The common feature among these cytoskeletal integrators is the presence of tandem arrays of units known as spectrin repeats (SRs). In addition, many of these proteins have calponin-homology $(\mathrm{CH})$ domains that bind to actin filaments. Other features found in various subclasses of these cytoskeletal integrators are domains that bind to microtubules, intermediate filaments, and the nuclear membrane. Furthermore, there are other proteinprotein interaction domains in most of these molecules. First, I will discuss the simplest of these proteins- $\alpha$-actinin-followed by spectrin and dystrophin/utrophin, be- fore considering the spectraplakin/plakin members, and finally the nesprin proteins.

\section{$2 \alpha$-ACTININ}

$\alpha$-actinin is known primarily as an actin-cross-linking protein and has been reviewed in this capacity elsewhere (Pollard 2016). It is included in this review of cytoskeletal integrators because it appears to be the ancestor protein to the family of proteins described here (Broderick and Winder 2005). $\alpha$-actinin comprises three domains, an actin filament-binding domain consisting of two $\mathrm{CH}$ domains, a series of four SRs, and a domain consisting of EF handlike motifs (see Fig. 1A). As these domains are also present in the other cytoskeletal integrators discussed herein, they are described in more detail below.
A

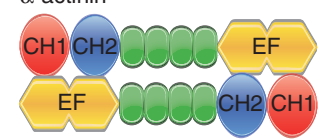

$\beta$-spectrin

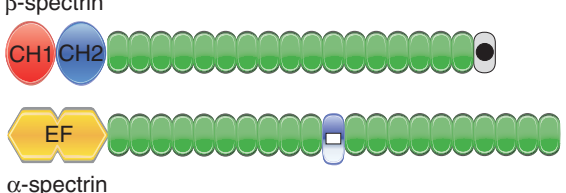

$\alpha$-spectrin

Dystrophin

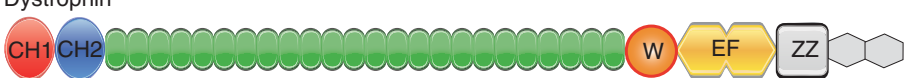

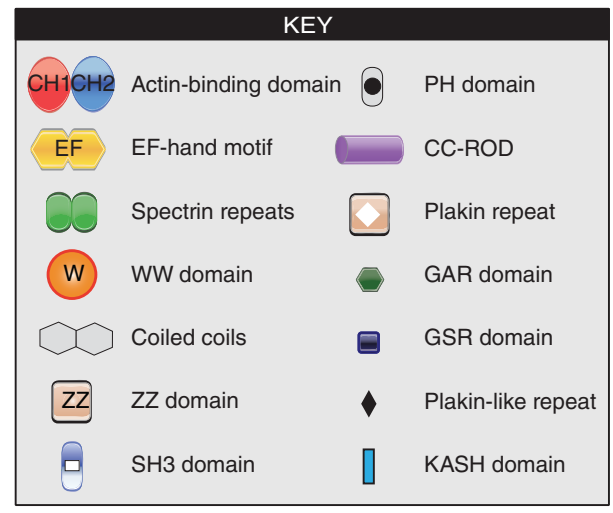

Utrophin

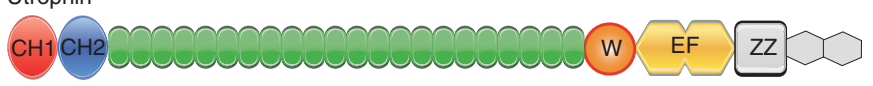

B Shot I

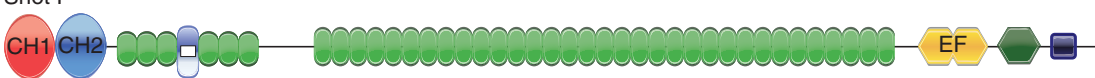

Shot II

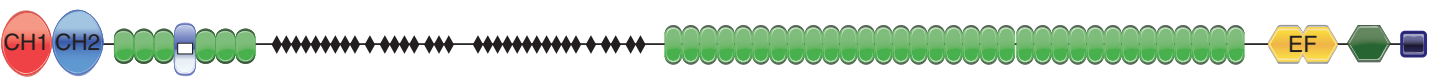

VAB-10A

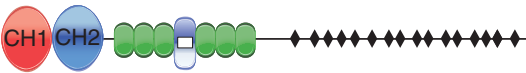

VAB-10B

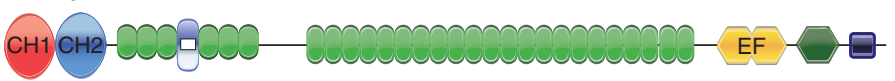

Figure 1. Schematic diagrams of the spectrin superfamily of cytoskeletal linker proteins. $(A) \alpha$-actinin, spectrin, and dystrophin/utrophin. $\alpha$-actinin is shown as an antiparallel dimer. The two actin-binding domains (ABDs) $\mathrm{CH} 1$ and $\mathrm{CH} 2$ allow the cross-linking of actin filaments. Spectrin is shown as a dimer of $\alpha$ - and $\beta$-spectrin, with $\alpha$-spectrin shown below $\beta$-spectrin in an antiparallel orientation (from carboxyl to amino terminus). A tetramer can be formed by a second antiparallel dimer in the opposite direction, with the amino terminus of $\alpha$-spectrin interacting with the carboxyl terminus of $\beta$-spectrin (Broderick and Winder 2005). The resulting tetramer resembles $\alpha$-actinin, but has many more spectrin repeats (SRs). Dystrophin is shown as a monomer. It can interact with actin through the ABD, but has many more interaction domains, including a second ABD in the last two SRs. Utrophin is similar to dystrophin, except that the last two SRs are missing. (Figure and legend continue on facing page.) 


\subsection{CH Domains}

The actin-binding domain (ABD) of the spectrin family of proteins consists of two calponin-homology $(\mathrm{CH} 1$ and $\mathrm{CH} 2$ ) domains in tandem. These ABDs are found near the amino termini of these proteins. Calponin, which is a calcium-binding protein present in smooth muscle, has a single $\mathrm{CH}$ domain that is not able to bind to actin. It is the prototype $\mathrm{CH}$ domain because it was first found in calpo- nin, where it was identified before it was found to be in actin-binding proteins. The $\mathrm{CH} 1$ domain is able to bind to actin filaments by itself, albeit with lower affinity than the combined $\mathrm{CH} 1-\mathrm{CH} 2$ domains (Gimona and Winder 1998). In contrast, the $\mathrm{CH} 2$ domain alone cannot bind to actin filaments. None of the members of the spectrin superfamily expresses only the $\mathrm{CH} 1$ domain, although there are isoforms that express only the $\mathrm{CH} 2$ domain (Jefferson et al. 2004). Structurally, the $\mathrm{CH}$ domain is a compact globular

\section{C}
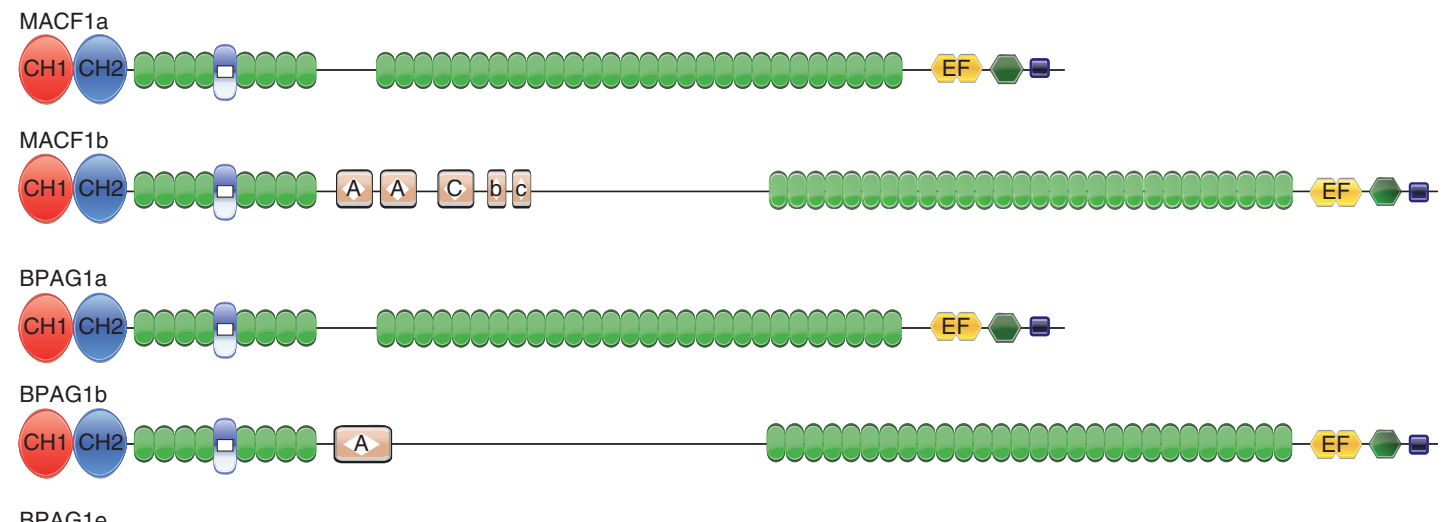

BPAG1e
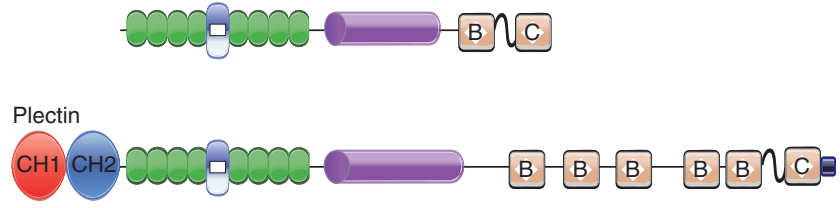

Desmoplakin I

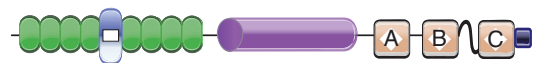

D
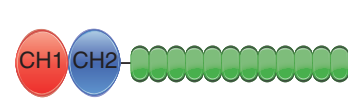

Nesprin-1G

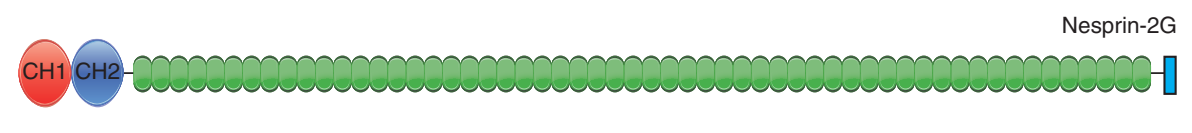

Nesprin-3 $\alpha$

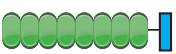

Nesprin-4

Figure 1. (Continued) The domain labeled "ZZ" is the zinc-finger domain. (B) Invertebrate spectraplakins. The two splice forms of the two known invertebrate spectraplakins are shown. Shots I and II are the Drosophila spectraplakins, and VAB-10a and VAB-10b are the Caenorhabditis elegans spectraplakins. The identity of the various domains is given in the key. $(C)$ Vertebrate spectraplakins and plakins. The major "a" and "b" splice forms of microtubule-actin crosslinking factor 1 (MACF1) and bullous pemphigoid antigen 1 (BPAG1) are shown. MACF1a and MACF1b have domain structures similar to those of BPAGla and BPAG1b, respectively. However, a third splice form of BPAG1BPAG1e-only shares the so-called plakin domain in common with the other two isoforms. BPAGle can dimerize through its coiled-coil rod. Plectin and desmoplakin, along with BPAGle, are considered "plakins" rather than "spectraplakins," although the plakin domain actually consists of SRs. (D) Nesprins. The four major isoforms of the nesprins are shown. There are some unusual SRs in the two giant isoforms, but, for simplicity, they are shown here as standard SRs. 
domain that comprises four $\alpha$-helices that are 11-18 residues in length and show a roughly parallel orientation, interspersed with three shorter helices (Broderick and Winder 2002).

\subsection{SR Domains}

SRs occur in tandem and constitute the so-called rod domain or SR domain. This domain is required for proteins to be considered members of the spectrin superfamily of cytoskeletal integrators. These SR domains consist of between 2 and 74 SRs and act as flexible spacers between the actin cytoskeleton and the membrane or other cytoskeletal elements. In addition, the SR domain can function to interact with other molecules in the cytoplasm. The repeats are 106-122 residues in length. Each repeat comprises three $\alpha$-helices, and tandem SRs are connected with an interspectrin repeat that is also helical in nature (Fig. 2).

Proteins with spectrin repeat-containing rod domains can dimerize in either a parallel or antiparallel manner. SRs can have limited homology at the amino acid level but are related structurally. Protein-prediction programs can identify SRs based on the expected secondary structure.

\subsection{EF Hands}

The carboxyl terminus of $\alpha$-actinin has an EF hand motif. The EF hand consists of two $\alpha$-helices linked by a short loop region. This helix-loop-helix arrangement resembles the spread thumb and forefinger of a hand. Calcium ions can bind to the EF hand, leading to a conformational change (Tufty and Kretsinger 1975), although a number of EF hand-containing molecules have lost their ability to bind calcium. This motif is present in members of the spectrin superfamily, but it is not a defining motif of this family.

\subsection{Structure and Function of $\alpha$-Actinin}

$\alpha$-actinin comprises the three structural motifs described above (Fig. 1A). There are two $\mathrm{CH}$ domains at the amino terminus, followed by a rod domain with four SRs and an EF hand motif. The rod domains of two $\alpha$-actinin molecules dimerized in an antiparallel fashion allow the molecule to serve as an actin filament-cross-linking protein (Fig. 1A). The small number of SRs results in a short spacer in the cross-linked actin filaments. The EF hand allows regulation of actin binding, but not all $\alpha$-actinin isoforms have EF hands that can chelate calcium.

\section{SPECTRINS}

Spectrin was first identified as the major component of the erythrocyte membrane cytoskeleton. It has since been

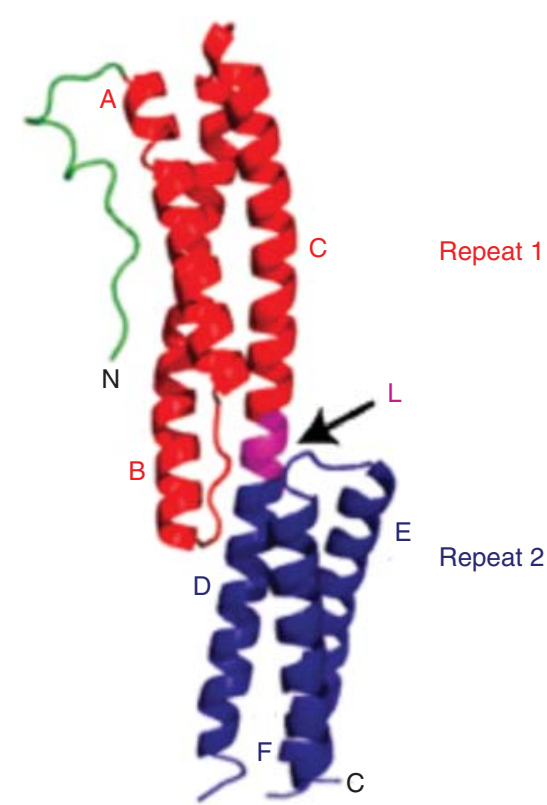

Figure 2. Ribbon diagram illustrating the structure of two spectrin repeats (SRs) in the plakin domain of bullous pemphigoid antigen 1 (BPAG1/dystonin), which is a member of the spectrin superfamily. Depicted is a loop-like region (green) at the far amino-terminal end, followed by a pair of SRs 1 and 2 (red and blue, respectively) arranged in tandem and connected by a linker region ( $\mathrm{L}$, purple) that is also helical in nature. Also labeled are the component helices $\mathrm{A}-\mathrm{C}$ and D-F. (Reprinted from Jefferson et al. 2004.)

found to be a common component that lines the intracellular side of the plasma membrane of cells of many vertebrate tissues and is also present in a number of nonvertebrates. Spectrin was named as such because it was found to be the major component of so-called red cell "ghosts," which are the remnants of the blood cells after treatment with mild detergent to release hemoglobin and other cytoplasmic components. Spectrin comprises $\alpha$ - and $\beta$-subunits that interact in an antiparallel manner to form an $\alpha \beta$ dimer (Fig. 1A). Two dimers associate to form a tetramer. Formation of a tetramer occurs through the interaction of the amino terminus of the $\alpha$-subunit with the carboxyl terminus of the $\beta$-subunit (Broderick and Winder 2005). The spectrin tetramer is present at the internal side of the plasma membrane, where it interacts with integral membrane proteins, as well as with the actin cytoskeleton.

In mammals, two genes encode the $\alpha$-spectrins, and five genes encode the $\beta$-spectrins. Red blood cell spectrin consists of the $\alpha \mathrm{I}$ and $\beta \mathrm{I}$ isoforms. Nonerythrocyte $\alpha$-spectrin is encoded by several isoforms, together with combinations of $\beta \mathrm{II}, \beta \mathrm{III}$, and $\beta \mathrm{IV}$ isoforms. Low levels of $\beta$ V are present in many tissues as well.

The $\alpha \beta$-spectrin dimer contains elements similar to those of $\alpha$-actinin (Fig. 1A). Broderick and Winder 
(2005) described the evolutionary progression from $\alpha$-actinin to $\alpha \beta$-spectrin, with the elongation of $\alpha$-actinin by the addition of SRs, duplication leading to an $\alpha \beta$ ancestor, and subsequent separation into $\beta$-chains and $\alpha$-chains, with $\beta$-spectrin corresponding to the amino terminus and $\alpha$-spectrin to the carboxyl terminus of the ancestral $\alpha$-actinin. Thus, $\beta$-spectrin consists of $\mathrm{CH} 1$ and $\mathrm{CH} 2$ domains followed by SRs, whereas $\alpha$-spectrin consists of SRs followed by an EF hand. The spectrin tetramer therefore has functional domains similar to those of the $\alpha$-actinin dimer. In addition, the first two SRs of $\beta$-spectrin and the last two SRs of $\alpha$-spectrin are more closely related to the first two and last two SRs of $\alpha$-actinin, respectively.

Spectrin is the major component of the membrane skeleton in the erythrocyte, which allows the cell to respond to strong mechanical stresses, including high shear stress during blood circulation and shape changes when it passes through small capillaries. Spectrin binds to the erythrocyte membrane through two major protein complexes. The $\mathrm{ABD}$, comprising two $\mathrm{CH}$ domains, allows spectrin to bind to actin, as well as band 4.1. There are a number of accessory proteins involved in the spectrin-actin interaction complex at the membrane. $\beta$-spectrin also binds to ankyrin through a region on the 15th to 16th SR. Ankyrin mediates the interaction between spectrin and other transmembrane proteins. There are additional protein-interaction domains on spectrin, including the Src-homology 3 (SH3) domain on $\alpha$-spectrin, as well as a pleckstrin-homology $(\mathrm{PH})$ domain at the carboxyl terminus of $\beta$-spectrin. The $\mathrm{PH}$ domains are present in a number of proteins and play a role in cellular signaling, as well as in cytoskeletal organization. Finally, spectrin is thought to form a large platform of interactions with numerous binding sites, leading to the idea that SRs can function as a docking surface for signal transduction and integral membrane proteins (Djinovic-Carugo et al. 2002).

\section{DYSTROPHIN AND UTROPHIN}

\subsection{Dystrophin}

Mutations in dystrophin cause Duchenne muscular dystrophy, an X-linked muscle-wasting disease. The structure of dystrophin resembles that of $\alpha$-actinin in that it contains an actin-binding motif consisting of $\mathrm{CH} 1$ and $\mathrm{CH} 2$ domains in tandem, SRs, and an EF hand (Fig. 1A) (Broderick and Winder 2005). However, dystrophin has 24 SRs, as opposed to the four repeats of $\alpha$-actinin. Following the 24th repeat, there is a cysteine-rich domain, which contains a WW domain, a protein binding module similar to the $\mathrm{SH} 3$ domain that is capable of binding proline-rich molecules, two EF hand motifs, again similar to $\alpha$-actinin, followed by a zinc-finger domain ("ZZ" in Fig. 1A). Finally, at the carboxyl terminus, there is an $\alpha$-helical domain that binds to cytosolic components.

Dystrophin is present at the muscle sarcolemma, where it acts as a cytoskeletal integrator, linking the actin cytoskeleton to a membrane-spanning complex (dystrophinassociated glycoprotein complex) that interacts with the basement membrane. Specifically, the cysteine-rich domain interacts with $\beta$-dystroglycan, a part of this complex, whereas the $\alpha$-helical domain binds to dystrobrevin. The SR domain of dystrophin also contains a second actinbinding site unrelated to the amino-terminal $\mathrm{CH}$ domain-containing ABD. Duchenne muscular dystrophy is usually caused by mutations in dystrophin that cause early termination, resulting in the absence of the interaction domains at the carboxyl terminus of the molecule. In Becker muscular dystrophy, in-frame deletions cause a milder form of the disease. These deletions can shorten the dystrophin molecule, but leave the carboxy-terminal regions intact. In addition to forming a link between the actin cytoskeleton and the membrane, the gene encoding dystrophin, like many other molecules described in this review, has multiple promoters and translational start sites. These alternative start sites do not affect the various domains described here, but enable tissue-specific expression of the new isoforms possessing alternative amino-terminal regions that could impart slightly different functions.

\subsection{Utrophin}

Utrophin is a dystrophin-related protein that is more ubiquitously expressed than dystrophin, but shares a high degree of functional and structural similarity (Fig. 1A) (Broderick and Winder 2005). Its carboxyl terminus can also interact with dystroglycan, and it is up-regulated in patients with Duchenne muscular dystrophy. Utrophin contains 22 SRs and binds to actin through its amino-terminal ABD. Although the general domain organization of dystrophin and utrophin resembles that of $\alpha$-actinin, the homology between the SRs among $\alpha$-actinin and spectrin is much higher than between $\alpha$-actinin and dystrophin, suggesting that dystrophin did not simply evolve from $\alpha$ actinin by duplication of the SRs.

\section{SPECTRAPLAKINS AND PLAKINS}

Although they are often categorized separately, the spectraplakins and plakins are actually part of the same family (Sonnenberg and Liem 2007; Suozzi et al. 2012). Plakins were first identified as part of the complex that tethers the keratin intermediate filaments to cell-cell and cell-matrix junctional complexes (Leung et al. 2002; Jefferson et al. 2004). The spectraplakins were named as such because 
they appeared to comprise a hybrid of spectrin and plakins (Roper and Brown 2003). The spectraplakins have probably evolved from the spectrins and play a general role in interacting with the microtubule and actin cytoskeletal networks. They have subsequently evolved to the so-called plakins that are involved in intermediate-filament junctional complexes. The bullous pemphigoid antigen 1 (BPAG1) gene (called DST) is alternatively spliced to form both spectraplakin and plakin isoforms (Leung et al. 2001).

\subsection{Invertebrate Spectraplakins}

Drosophila has a single spectraplakin locus, called short stop or shot. short stop was first described in a screen for mutations that caused defects in neuromuscular connectivity. short stop was identified as a mutation in which motor neurons failed to reach their target. It was found to be allelic to another mutation-kakapo - that was found in a screen to isolate genes required for integrin-mediated adhesion (Gregory and Brown 1998; Lee et al. 2000). The shot gene yields two major isoforms related to the spectrin family. ShotI consists of two $\mathrm{CH}$ domains followed by a series of SRs (Fig. 1B). The SRs are organized in two distinct groups-one resembles the "plakin" domain that was considered to be the signature domain of the entire plakin family of proteins described below. This plakin domain is now known to consist of an SH3 domain flanked on each side by three (or possibly four) SRs (Sonnenberg and Liem 2007). Amino acid homology did not immediately identify this domain as containing SRs, but X-ray studies of this domain of three different plakins have shown that they are SRs (Jefferson et al. 2007; Sonnenberg et al. 2007; Choi and Weiss 2011).

The second group consists of a stretch of 33 SRs comprising the rod domain. At the carboxyl terminus of ShotI are two putative EF hands followed by a domain that binds to microtubules. The microtubule-binding domain was first described for the vertebrate spectraplakin microtubule-actin cross-linking factor 1 (MACF1) (see below) and is a unique domain that consists of a gas2-related domain and a domain that contains glycine-serine-arginine repeats (Leung et al. 1999). Each of these domains can bind to microtubules. shot therefore has most of the domains found in a combination of $\alpha$ - and $\beta$-spectrin, with the added ability to bind to microtubules. The microtubuleactin cross-linking function of ShotI is important for axon extension as this property can be rescued by a construct that consists of the actin- and microtubule-binding domains expressed in the neurons of shot mutant embryos (Lee and Kolodjiez 2002). Shot also has a function in cell adhesion as it is necessary for apical surface remodeling during tracheal tube fusion. The carboxyl terminus of Shot is also able to bind microtubule-associated end-binding protein EB1 to promote microtubule assembly. The second alternatively spliced isoform (ShotII) has a large insert with a series of repeats, the function of which has not been elucidated.

The nematode Caenorhabditis elegans also has a single spectraplakin/plakin gene called vab-10 (Bosher et al. 2003). Like Shot, there are two alternatively spliced forms of VAB-10 (Fig. 1B). VAB-10b resembles ShotI in its domain structure, whereas VAB-10a resembles part of ShotII; it has the $\mathrm{CH}$ domains and the plakin domain, as well as the repeats found in Shot II, but not the second SR domain and microtubule-interacting domain. This isoform has a similarity to the vertebrate plakin, plectin, described below. The two isoforms have different functionslack of VAB-10a results in the detachment of the epidermis from the cuticle and muscle, whereas lack of VAB-10b results in increased epidermal thickness. Whether there are effects on axonal outgrowth has not been reported.

\subsection{Vertebrate Spectraplakins}

In the following I describe the major vertebrate spectraplakins/plakins and give an account of how plakins are believed to have evolved from the spectraplakins. Also, I briefly describe the other members of the spectraplakin/ plakin family.

\subsubsection{MACF1}

MACF1 is also known as actin control factor 7 (ACF7), macrophin (Okuda et al. 1999), and $\alpha$-trabeculin (Sun et al. 1999). MACF1 is one of two spectraplakin genes identified in vertebrates (Leung et al. 2002; Jefferson et al. 2004; Sonnenberg and Liem 2007). Like invertebrate spectraplakins, MACF1 is alternatively spliced into two major isoforms, MACF1a and MACF1b (Lin et al. 2005). As can be seen in Figure 1c, MACF1a is very similar in its domain structure to ShotI and VAB-10b. The amino terminus of MACF1a contains $\mathrm{CH} 1$ and $\mathrm{CH} 2$ domains and can bind to actin. An amino-terminal fragment of MACF1 was first identified by a polymerase chain reaction strategy designed to identify members of the $\alpha$-actinin, dystrophin, and spectrin families (Byers et al. 1995). The resulting cDNA encoded the $\mathrm{CH}$ domains and the plakin domain. As noted above, the plakin domain actually consists of an $\mathrm{SH} 3$ domain flanked by SRs.

Like ShotI and VAB10b, MACF1a has a second SR domain (with 28 SRs) followed by the carboxy-terminal microtubule-binding domains described above for ShotI. Thus, MACF1 cross-links the actin and microtubule cytoskeletons. Partners that bind to the SH3 domain of MACF1 have not yet been identified. Mice that are null for Macf1 
die at the gastrulation stage (Chen et al. 2006). In cultured fibroblasts from $\mathrm{Macf1}^{-}{ }^{-}$mice, microtubules no longer grow along polarized actin-rich cortical structures in the absence of MACF1, consistent with its function as a microtubule-actin cross-linker (Kodama et al. 2003). Duplication in this gene has recently been described as causing a novel neuromuscular disease (Jørgensen et al. 2015).

MACF1 also regulates the cortical localization of the microtubule-tracking protein "CLIP-associating protein 2" and, like ShotI, can interact with EB1, suggesting further roles of this protein for microtubule dynamics and stability. Conditional knockout of MACF1 in the epidermis compromises the targeting of microtubules along F-actin to focal adhesions and impairs epidermal migration ( $\mathrm{Wu}$ et al. 2011). The nervous system-specific knockout of Macf1 leads to defects in the migration of neurons in traversing the cortical plate of the brain (Goryunov et al. 2010; $\mathrm{Ka}$ et al. 2014). These data suggest a crucial role for MACF1 in neuronal migration that is dependent on its ability to interact with both microfilaments and microtubules. MACF1-deficient neurons showed reduced density and aberrant morphology of dendritic spines (Ka and Kim 2015). The conditional knockout also revealed a third MACF1 isoform starting before the plakin domain, although the exact start site has not been identified (Goryunov et al. 2010). Other isoforms of MACF1, with different amino and carboxyl termini, have also been identified. Like ShotII, MACF1b has a series of repeats that resemble the repeats found at the carboxyl termini of the plakins, and these have been called plakin or plectin repeats. The interaction partners of this repeat region in MACF1b have not been identified.

\subsubsection{BPAG1}

BPAG1, also known as dystonin, is the second mammalian spectraplakin and is encoded by the gene DST. The alternative spliced forms of BPAG1 show the clear relationship between the so-called spectraplakins and plakins (Fig. 1C) as BPAG1a/b isoforms closely resemble MACF1a/b, ShotI, and VAB-10b. A third major isoform, BPAGle, resembles the plakins that link intermediate filaments to junctional complexes. The only domain in common with BPAGla and BPAG1b is the plakin domain (Leung et al. 2001). BPAG1e (also known as BP230) was identified before BPAGla and BPAG1b as one of two autoantigens present in the inflammatory skin-blistering disease bullous pemphigoid. BPAG1e and the other autoantigen (BPAG2) are components of the hemidesmosome in the epidermis and form a link between the keratin intermediate filaments and $\alpha 6 \beta 4$ integrin that, in turn, binds to laminin in the extracellular matrix (Jefferson et al. 2004).
BPAGla has a domain structure identical to that of MACF1a, as well as to that of ShotI. BPAG1b has domains similar to those of MACF1b. BPAG1a is expressed in the nervous system, where its main function is thought to be to cross-link microtubules and actin filaments. Bpag1 ${ }^{-/-}$ mice show sensory neuron degeneration and are identical to a previously described mutant mouse called dystonia musculorum (helping to explain the alternative name, dystonin) (Brown et al. 1995; Yang et al. 1996). For the sake of simplicity, this review refers to the protein and its isoforms as BPAG1. Although the basic domain structures of BPAGla and MACF1a are similar, the two proteins are not completely redundant as removal of MACF1 is not compensated by the presence of BPAG1. In contrast, the phenotype of Bpag1 ${ }^{-/-}$mice is mostly limited to sensory neurons, although the protein is present throughout the nervous system, suggesting that, in some cells, MACF1 might compensate for the loss of BPAG1. The function(s) of BPAG1b are still not fully known, except that this isoform is the predominant isoform present in muscle.

The basic domain structure of BPAG1e is similar to that of desmoplakin and plectin, as described below. As can be seen in Figure 1C, the only domain that BPAGle has in common with BPAGla/b is the plakin domain (Leung et al. 2001). After this domain, BPAGle has completely different domains, including a coiled-coil rod domain that allows it to dimerize and a plakin (or plectin) repeat-containing domain (PRD) at its carboxyl terminus. The PRD binds to intermediate filaments, although the binding site for intermediate filaments is in the linker regions between the plakin repeats. Plakin repeats are also present in all the other plakins that interact with intermediate filaments and junctional complexes. BPAGle is expressed in the basal layer of the epidermis, where it links the keratin intermediate filaments with hemidesmosomes, structures that are present at the interface between the basal cells and the extracellular matrix. The interaction site of BPAG1e with $\alpha 6 \beta 4$ integrin is within the amino-terminal region. BPAGle has a translation initiation site after the $\mathrm{CH}$ domains. This isoform is missing the $\mathrm{ABD}$ and, therefore, cannot bind to actin filaments. Instead, BPAGle is part of the cross-bridge between intermediate filaments and the cell junction. Although BPAGle links keratin filaments to the hemidesmosomes, Bpag1 ${ }^{-/-}$mice have only a minor skin phenotype, suggesting that other proteins in the skin might compensate for the absent protein.

\subsubsection{Plectin}

Plectin is also a component of hemidesmosomes, in which, like BPAG1e, it forms the link between the keratin intermediate filaments and the junctions of the basal cells with 
the extracellular matrix (Wiche 1989). It has a structure similar to that of BPAG1e, except that it also contains $\mathrm{CH}$ domains that can bind to actin filaments (Fig. 1C). Following the $\mathrm{CH}$ domains, plectin also has a coiled-coil rod that allows it to dimerize and a series of plakin repeats. The site allowing binding to intermediate filaments is in the carboxy-terminal PRD. Therefore, plectin can also link actin filaments to intermediate filaments. Plectin has also been reported to be able to bind to microtubules, but it does not have microtubule-binding domains similar to those of ShotI, MACF1a/b, or BPAGla/b. Plectin binds to $\alpha 6 \beta 4$ integrin in hemidesmosomes, along with BPAG1e. However, unlike Bpag1-/- mice, plectin ${ }^{-/-}$ mice show skin blistering. Plectin has multiple translation initiation sites that confer tissue specificity as plectin is found in a number of different tissues. In striated muscle, plectin is a component of the Z-line, in which it associates with the intermediate filament protein desmin. Absence of plectin can lead to misaligned myofibers, focal disruptions of sarcomeres, and rupture of the sarcolemma (Andra et al. 1997).

\subsubsection{Desmoplakin}

Desmoplakin is the sole plakin found in desmosomal complexes - the junctions that are found between cells in the epidermis (Green and Bornslaeger 1999). Desmoplakin has a structure similar to that of BPAGle, with an aminoterminal spectrin repeat-containing plakin domain followed by a coiled-coil rod domain that allows the molecule to dimerize and a PRD that interacts with intermediate filaments (Fig. 1C). The amino-terminal plakin domain has the interaction sites for the desmosomal cadherins found at desmosomes. Other molecules also locate to this complex, including plakoglobin ( $\gamma$-catenin) and plakophilins 1 and 2. Thus, desmoplakin plays a crucial role in linking the intermediate filament cytoskeleton to junctional complexes. Desmoplakin-null mice are embryonic lethal. In addition, posttranslational modifications of desmoplakin have been shown to play an essential role in coordinating cytoskeletal dynamics and cellular adhesion (Albrecht et al. 2015).

\subsubsection{Additional Plakin Family Members}

BPAGle, plectin, and desmoplakin are generally called plakins rather than spectraplakins. Plakins were considered to be linker proteins between cell junctions and the intermediate filament cytoskeleton. They appeared to differ from the spectraplakins in the absence of SRs. However, the clear identification of SRs in the plakin domains of these proteins (Jefferson et al. 2007; Sonnenberg et al. 2007; Choi and Weiss 2011) places these proteins as part of the spectrin superfamily. There are two additional plakin proteins with plakin domains-periplakin and envoplakin. There is also one protein that contains only PRDs, but no plakin domain, called epiplakin (Sonnenberg and Liem 2007).

\section{NESPRINS}

The spectrin family members described above link the cytoskeleton to themselves, the cell membrane, and junctional complexes. Nesprins are the most recently described members of this family and allow for interactions of the cytoskeleton with the nucleus (Warren et al. 2005). There are four nesprin ("nuclear envelope spectrin repeat protein") proteins-nesprin-1-nesprin-4, along with various alternatively spliced forms - encoded by synaptic nuclear protein genes SYNE1-SYNE4. The four nesprins are illustrated in Figure 1D.

\subsection{Nesprin-1 and Nesprin-2}

Nesprin-1 and -2 are the "giant" nesprins (nesprin-1G and nesprin-2G), possessing molecular masses of $\sim 1000 \mathrm{kDa}$ and $\sim 800 \mathrm{kDa}$, respectively (Chang et al. 2015). Alternative splicing of each of the two corresponding SYNE-1 and SYNE-2 genes leads to the synthesis of approximately $10-$ 20 isoforms. Like many members of the SR family, nesprin$1 \mathrm{G}$ and $-2 \mathrm{G}$ have an $\mathrm{ABD}$ comprising $\mathrm{CH} 1$ and $\mathrm{CH} 2$ domains. A large number of SRs with weak homology with the SRs of dystrophin and spectrin follow the ABD. There are 76 and 56 SRs in nesprin-1G and $-2 \mathrm{G}$, respectively. At the carboxyl terminus of the nesprin molecules is a "klarsicht, ANC-1, Syne homology" (KASH) domain-a targeting motif that anchors the protein to the nuclear envelope. The KASH domain consists of a 21-aminoacid transmembrane region, followed by a sequence that reaches into the lumen located between the two nuclear envelope membranes. The KASH domain is part of the "linker of nucleoskeleton and cytoskeleton" (LINC) complex (Fig. 3), which also includes inner nuclear membrane SUN proteins, which are type II membrane proteins with a single transmembrane segment. The amino terminus of the SUN proteins faces the nucleoplasm, where it binds to type $\mathrm{V}$ intermediate filament proteins-the nuclear lamins-as well as to chromatin. Thus, nesprins form the bridge between the nucleoskeleton and the cytoskeleton.

\subsection{Nesprin-3}

Nesprin-3 comprises SRs, followed by the KASH domain. There are two isoforms: Nesprin-3a has eight SRs and nesprin-3b has an alternative start site, resulting in the absence of the first SR. Nesprin-3 does not contain $\mathrm{CH}$ domains, but the first SR of nesprin-3a is able to bind to the $\mathrm{CH}$ 


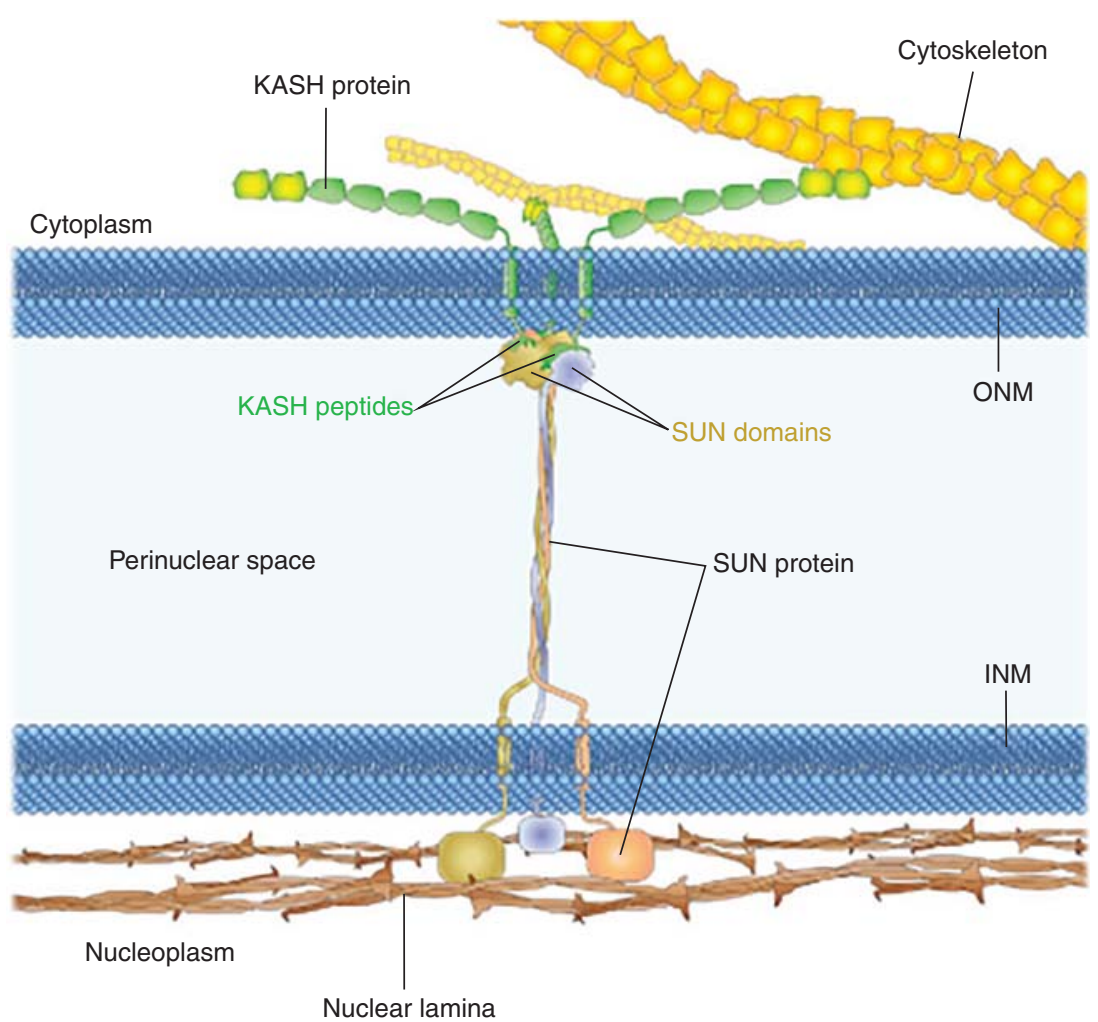

Figure 3. Depiction of the structure of the LINC (linker of nucleoskeleton and cytoskeleton) complex that bridges the cytoskeleton and nucleoskeleton. The complex comprises nesprin proteins ("KASH proteins") in the outer nuclear membrane (ONM) and SUN proteins in the inner nuclear membrane (INM). Extending into the cytoplasm are extensions of the nesprins that are of variable size and interact with different cytoskeletal elements. The SUN proteins anchor the LINC complex to the nucleoskeleton through their nucleoplasmic domains by means of interaction with the nuclear lamina, as well as with chromosome-binding proteins and probably other anchoring proteins. (Reprinted from Chang et al. 2015.)

domains of plectin and, through plectin, is indirectly bound to intermediate filaments (Ketema et al. 2013). This SR is also able to bind to the CH domains of BPAG1 and MACF1, and thereby nesprin-3a is capable of linking the nucleoskeleton to both cytoplasmic microtubules and intermediate filaments through these other linker proteins.

\subsection{Nesprin-4}

Nesprin-4 contains a KASH domain and just two SRs. It interacts with microtubules through an interaction with the microtubule motor protein kinesin. However, nesprin-4 is limited in its expression to secretory epithelia and the hair cells in the cochlea. Deletion of nesprin-4 in mice leads to progressive high-frequency hearing loss.

\section{CONCLUDING REMARKS}

Here, we have described the members of the spectrin superfamily that provide the links between the nucleus and the plasma membrane and to the cytoskeleton. The signature of this family of proteins is the SR that can be identified structurally, but is not necessarily conserved at the amino acid level. Each of the family members has multiple interaction domains, including domains that bind to the plasma membrane, the nucleoskeleton, and all the cytoskeletal elements. There are still possible interaction partners for all of these molecules that might be identified in the future that will help regulate cytoskeletal interactions, as well as cell adhesion. Recent studies have shown that posttranslational modifications of various domains can regulate these interactions. The importance of many of these family members in genetic diseases has been partially described, but we expect that additional family members will be shown to be involved in disease in the future.

\section{ACKNOWLEDGMENTS}

I thank Ms. Francine Delre for preparing Figure 1 for this review. 


\section{REFERENCES}

* Reference is in this collection.

Albrecht LV, Zheng L, Shabanowitz J, Purevjav E, Towbin JA, Hunt DF, Green KJ. 2015. GSK3- and PRMT-1 dependent modification of desmoplakin control desmoplakin-cytoskeleton dynamics. J Cell Biol 208: $597-612$.

Andra K, Lassmann H, Bittner R, Shorny S, Fässler R, Probst F, Wiche G. 1997. Targeted inactivation of plectin reveals essential function in maintaining the integrity of skin, muscle and heart cytoarchitecture. Genes Dev 11: 3143-3156.

Bosher JM, Hahn BS, Legouis R, Sookhareea S, Weimer RM, Gansmuller A, Chisholm AD, Rose AM, Bessereau JL, Labouesse M. 2003. The Caenorhabditis elegans vab-10 spectraplakin isoforms protect the epidermis against internal and external forces. J Cell Biol 161: 757-768.

Broderick MJ, Winder SJ. 2002. Towards a complete atomic structure of spectrin family proteins. J Struct Biol 137: 184-193.

Broderick MJ, Winder SJ. 2005. Spectrin, $\alpha$-actinin, and dystrophin. $A d v$ Protein Chem 70: 203-246.

Brown A, Bernier G, Mathieu M, Rossant J, Kothary R. 1995. The mouse dystonia musculorum gene is a neural isoform of bullous pemphigoid antigen 1. Nat Genet 10: 301-306.

Byers TJ, Beggs AH, McNally EM, Kunkel LM. 1995. Novel actin crosslinker superfamily member identified by a two step degenerate PCR procedure. FEBS Lett 368: 500-504.

Chang W, Worman HJ, Gundersen GG. 2015. Accessorizing and anchoring the LINC complex for multifunctionality. J Cell Biol 208: $11-22$.

Chen HJ, Lin CM, Lin CS, Perez-Olle R, Leung CL, Liem RK. 2006. The role of microtubule actin cross-linking factor 1 (MACF1) in the Wnt signaling pathway. Genes Dev 20: 1933-1945.

Choi HJ, Weiss WI. 2011. Crystal structure of a rigid four-spectrin repeat fragment of the human desmoplakin plakin domain. J Mol Biol 409: $800-812$.

Djinovic-Carugo K, Gautel M, Ylanne J, Young P. 2002. The spectrin repeat: A structural platform for cytoskeletal protein assemblies. FEBS Lett 513: 119-123.

Gimona M, Winder SJ. 1998. Single calponin homology domains are not actin-binding domains. Curr Biol 8: R674-R675.

Goryunov D, He CZ, Lin CS, Leung CL, Liem RK. 2010. Nervous-tissuespecific elimination of microtubule-actin crosslinking factor la results in multiple developmental defects in the mouse brain. Mol Cell Neurosci 44: 1-14.

Green KJ, Bornslaeger EA. 1999. Desmoplakin. In Guidebook to the extracellular matrix, anchor, and adhesion proteins, 2nd ed. (ed. Kreis T, Vale R), pp. 103-105. Oxford University Press, Oxford.

Gregory SL, Brown NH. 1998. kakapo, a gene required for adhesion between and within cell layers in Drosophila, encodes a large cytoskeletal linker protein related to plectin and dystrophin. J Cell Biol 143: $1271-1282$.

Jefferson JJ, Leung CL, Liem RK. 2004. Plakins: Goliaths that link cell junctions and the cytoskeleton. Nat Rev Mol Cell Biol 5: 542-553.

Jefferson JJ, Ciatto C, Shapiro L, Liem RK. 2007. Structural analysis of the plakin domain of bullous pemphigoid antigen1 (BPAG1) suggests that plakins are members of the spectrin superfamily. J Mol Biol 366: $244-257$.

Jørgensen LH, Mosbech MB, Færgeman NJ, Graakjaer J, Jacobsen SV, Schrøder HD. 2015. Duplication in the microtubule-actin crosslinking factor 1 gene causes a novel neuromuscular condition. Sci Rep 4: 5180.

Ka M, Kim WY. 2015. Microtubule-actin crosslinking factor 1 is required for dendritic arborization and axon outgrowth in the developing brain. Mol Neurobiol doi: 10.1007/s12035-015-9508-4.
Ka M, Jung EM, Mueller U, Kim WY. 2014. MACF1 regulates the migration of pyramidal neurons via microtubule dynamics and GSK-3 signaling. Dev Biol 395: 4-18.

Ketema M, Kreft M, Secades P, Janssen H, Sonnenberg A. 2013. Nesprin-3 connects plectin and vimentin to the nuclear envelope of Sertoli cells but is not require for Sertoli cell function in spermatogenesis. Mol Biol Cell 24: 2454-2466.

Kodama A, Karakesisoglou I, Wong E, Vaezi A, Fuchs E. 2003. ACF7: An essential integrator of microtubule dynamics. Cell 115: 343-354.

Lee S, Kolodjiez PA. 2002. Short stop provides an essential link between F-actin and microtubules. Development 129: 1195-1204.

Lee S, Harris KL, Whitington PM, Kolodziej PA. 2000. short stop is allelic to kakapo, and encodes rod-like cytoskeletal-associated proteins required for axon extension. J Neurosci 20: 1096-1108.

Leung CL, Sun D, Zheng M, Knowles DR, Liem RK. 1999. Microtubule actin cross-linking factor (MACF). A hybrid of dystonin and dystrophin that can interact with the actin and microtubule cytoskeletons. $J$ Cell Biol 147: 1275-1286.

Leung CL, Zheng M, Prater SM, Liem RKH. 2001. The BPAG1 locus: Alternative splicing produces multiple isoforms with distinct cytoskeletal linker domains, including predominant isoforms in neurons and muscles. J Cell Biol 154: 691-697.

Leung CL, Green KJ, Liem RK. 2002. Plakins: A family of versatile cytolinker proteins. Trends Cell Biol 12: 37-45.

Lin CM, Chen HJ, Leung CL, Parry DA, Liem RK. 2005. Microtubule actin crosslinking factor $1 \mathrm{~b}$ : A novel plakin that localizes to the Golgi complex. J Cell Sci 118: 3727-3738.

Okuda T, Matsuda S, Nakatsugawa S, Ichigotani Y, Iwahashi N, Takahashi M, Ishigaki T, Hamaguchi M. 1999. Molecular cloning of macrophin, a human homologue of Drosophila kakapo with a close structural similarity to plectin and dystrophin. Biochem Biophys Res Commun 264: 568-574.

* Pollard T. 2016. Actin and actin-binding proteins. Cold Spring Harb Perspect Biol doi: 10.1101/cshperspect.a018259.

Roper K, Brown NH. 2003. Maintaining epithelial integrity: A function for gigantic spectraplakin isoforms in adherens junctions. J Cell Biol 162: $1305-1315$.

Sonnenberg A, Liem RK. 2007. Plakins in development and disease. Exp Cell Res 313: 2189-2203.

Sonnenberg A, Rojas AM, de Pereda JM. 2007. The structure of a tandem pair of spectrin repeats of plectin reveals a modular organization of the plakin domain. J Mol Biol 368: 1379-1391.

Sun Y, Zhang J, Kraeft SK, Auclair D, Chang MS, Liu Y, Sutherland R, Salgia R, Griffin JD, Ferland LH, et al. 1999. Molecular cloning and characterization of human trabeculin- $\alpha$, a giant protein defining a new family of actin-binding proteins. J Biol Chem 274: 33522-33530.

Suozzi KC, Wu X, Fuchs E. 2012. Spectraplakins: Master orchestrators of cytoskeletal dynamics. J Cell Biol 197: 465-475.

Tufty RM, Kretsinger RH. 1975. Troponin and parvalbumin calcium binding regions predicted in myosin light chain and T4 lysozyme. Science 187: 167-169.

Warren DT, Zhang Q, Weissberg PL, Shanahan CM. 2005. Nesprins: Intracellular scaffolds that maintain cell architecture and coordinate cell function? Expert Rev Mol Med 7: 1-15.

Wiche G. 1989. Plectin: General overview and appraisal of its potential role as a subunit protein of the cytomatrix. Crit Rev Biochem Mol Biol 24: $41-67$.

Wu X, Shen QT, Oristian DS, Lu CP, Zheng Q, Wang HW, Fuchs E. 2011. Skin stem cells orchestrate directional migration by regulating microtubule-ACF7 connections through GSK3 $\beta$. Cell 144: $341-352$.

Yang Y, Dowling J, Yu QC, Kouklis P, Cleveland DW, Fuchs E. 1996. An essential cytoskeletal linker protein connecting actin microfilaments to intermediate filaments. Cell 86: 655-665. 


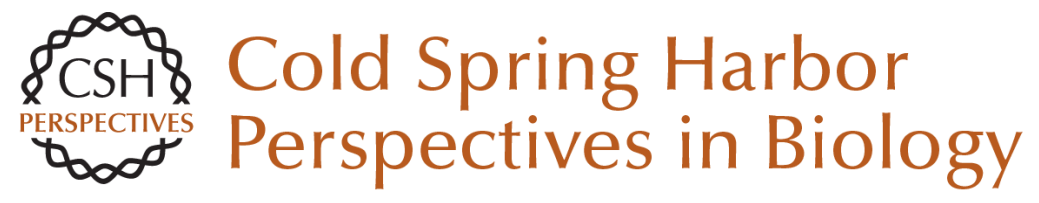

\section{Cytoskeletal Integrators: The Spectrin Superfamily}

Ronald K.H. Liem

Cold Spring Harb Perspect Biol 2016; doi: 10.1101/cshperspect.a018259

Subject Collection The Cytoskeleton

Microtubules and Microtubule-Associated

Proteins

Holly V. Goodson and Erin M. Jonasson

Motor Proteins

H. Lee Sweeney and Erika L.F. Holzbaur

Myosin-Driven Intracellular Transport Margaret A. Titus

The Actin Cytoskeleton and Actin-Based Motility Tatyana Svitkina

\section{Mechanical Properties of the Cytoskeleton and Cells \\ Adrian F. Pegoraro, Paul Janmey and David A.} Weitz

Intermediate Filaments and the Regulation of Cell Motility during Regeneration and Wound Healing Fang Cheng and John E. Eriksson

Intermediate Filaments and the Plasma Membrane Jonathan C.R. Jones, Chen Yuan Kam, Robert M. Harmon, et al.

Intracellular Motility of Intermediate Filaments Rudolf E. Leube, Marcin Moch and Reinhard Windoffer

\section{Overview of the Cytoskeleton from an}

Evolutionary Perspective

Thomas D. Pollard and Robert D. Goldman

Types I and II Keratin Intermediate Filaments Justin T. Jacob, Pierre A. Coulombe, Raymond Kwan, et al.

Muscle Contraction

H. Lee Sweeney and David W. Hammers

Type III Intermediate Filaments Desmin, Glial

Fibrillary Acidic Protein (GFAP), Vimentin, and

Peripherin

Elly M. Hol and Yassemi Capetanaki

Cytokinesis in Metazoa and Fungi Michael Glotzer

Ciliary Motility: Regulation of Axonemal Dynein Motors

Rasagnya Viswanadha, Winfield S. Sale and Mary

E. Porter

Actin-Based Adhesion Modules Mediate Cell Interactions with the Extracellular Matrix and Neighboring Cells

Alexia I. Bachir, Alan Rick Horwitz, W. James Nelson, et al.

Microtubule-Based Transport and the Distribution,

Tethering, and Organization of Organelles Kari Barlan and Vladimir I. Gelfand

For additional articles in this collection, see http://cshperspectives.cshlp.org/cgi/collection/

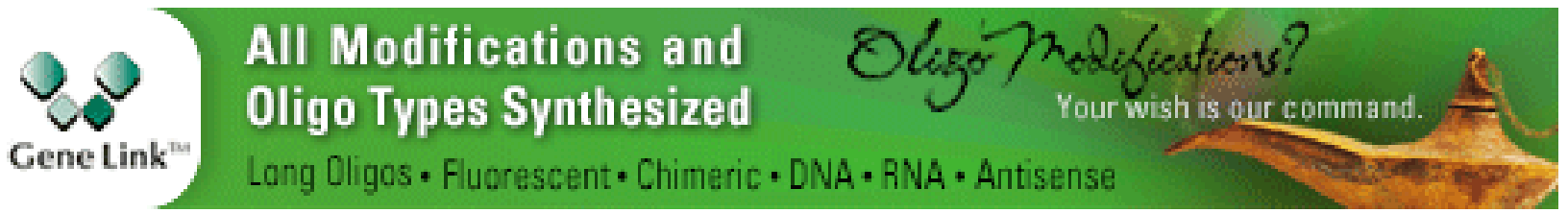

Copyright @ 2016 Cold Spring Harbor Laboratory Press; all rights reserved 
For additional articles in this collection, see http://cshperspectives.cshlp.org/cgi/collection/

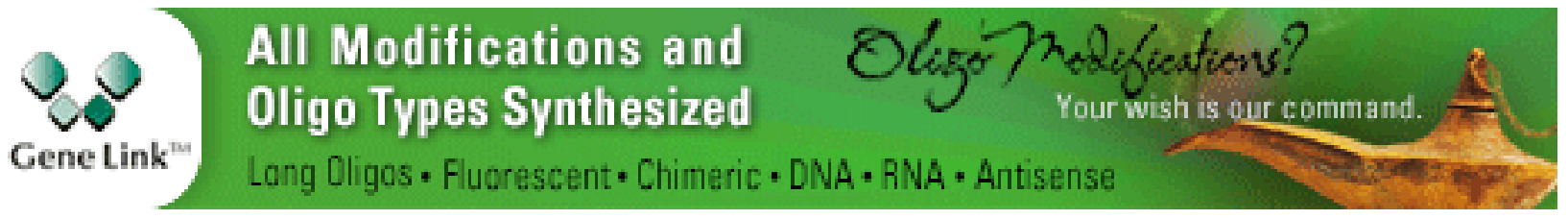

Copyright @ 2016 Cold Spring Harbor Laboratory Press; all rights reserved 